ULM-TP/00-8

December 2000, revised March 2001

\title{
Level spacings and periodic orbits
}

\author{
Stefan Keppeler \\ Abteilung Theoretische Physik, Universität Ulm, Albert-Einstein-Allee 11, D-89069 Ulm, Germany
}

\begin{abstract}
Starting from a semiclassical quantization condition based on the trace formula, we derive a periodic-orbit formula for the distribution of spacings of eigenvalues with $k$ intermediate levels. Numerical tests verify the validity of this representation for the nearest-neighbor level spacing $(k=$ $0)$. In a second part, we present an asymptotic evaluation for large spacings, where consistency with random matrix theory is achieved for large $k$. We also discuss the relation with the method of Bogomolny and Keating [Phys. Rev. Lett. 77 (1996) 1472] for two-point correlations.
\end{abstract}

The statistical distribution of quantum energy levels is conjectured to reflect the chaoticity or integrability of the underlying classical dynamics [1] 2]. For classically chaotic systems one expects spectral distributions like those computed in random matrix theory (RMT) [2], whereas for classically integrable systems the quantum levels appear to follow the distribution for a Poisson process [1]. Attempts to explain this correspondence are based on Gutzwiller's semiclassical trace formula [3] which bridges the gap between classical and quantum mechanics. The prime result in this direction is Berry's analysis of the spectral rigidity [4], based on the socalled diagonal approximation and the classical sum rule of Hannay and Ozorio de Almeida [5]. Starting from a semiclassical quantization condition [6, ], Bogomolny and Keating [8] were able to extract information on the two-point correlations going beyond the results of 䟣. It is the aim of the present work to follow a similar path in order to obtain semiclassical information on the level spacing distributions.

The Gutzwiller trace formula can be expressed as a periodic-orbit sum for the integrated density of states $N(E):=\sum_{n} \Theta\left(E-E_{n}\right)$,

$$
N_{T}(E) \sim \bar{N}(E)+\sum_{\left|r T_{\gamma}\right| \leq T} \sum^{\prime} \frac{\mathcal{A}_{\gamma r}}{2 \pi \mathrm{i} r} \mathrm{e}^{\frac{\mathrm{i}}{\hbar} r S_{\gamma}(E)},
$$

in the semiclassical limit, i.e. for $\hbar \rightarrow 0 . \bar{N}(E)$ denotes the mean part following from Weyl's law, whereas the fluctuations, $N_{T}^{\mathrm{fl}}(E)$, are given by a sum over all primitive periodic orbits $\gamma$ and their $r$-fold repetitions, the prime on the second sum indicating the exclusion of $r=0$. The (primitive) action and period of a periodic orbit are denoted by $S_{\gamma}(E)$ and $T_{\gamma}(E)$, respectively. The explicit form of the amplitudes $\mathcal{A}_{\gamma r}$ can be found in [3] for chaotic and in [9] for integrable systems. We have given a version truncated at periods $\left|r T_{\gamma}\right|=T$, which will be needed in the following. Instead of directly using (11) to express spectral functions, we will use an approximate spectrum $E_{n}(T)$ obtained from the condition [6], []

$$
N_{T}\left(E_{n}(T)\right) \stackrel{!}{=} n+\frac{1}{2} .
$$

Before investigating the statistical distribution we have to unfold the spectrum such that its mean density $\bar{d}(E)=$ $\mathrm{d} \bar{N}(E) / \mathrm{d} E$ is rescaled to unity. To this end, investigating spectral correlations in an interval $I(E ; \hbar):=$ $[E-\hbar \omega, E+\hbar \omega]$, we introduce the unfolded energies $x_{n}(T):=E_{n}(T) \bar{d}, \bar{d}:=\bar{d}(E)$, see, e.g., [10] for details. Spacings of two unfolded energies with $k$ intermediate levels are given by $s_{n}(k ; T)=x_{n+k+1}(T)-x_{n}(T)$ and integrated level spacing distributions are defined by

$$
I(k, s ; T):=\frac{1}{N_{I}} \sum_{E_{n} \in I(E ; \hbar)} \Theta\left(s-s_{n}(k ; T)\right),
$$

where $N_{I}$ denotes the number of eigenvalues contained in $I(E ; \hbar)$. The often used level spacing densities $P(k, s ; T)$ are the derivative of $I(k, s ; T)$ with respect to $s$. Since the condition $s \geq s_{n}(k ; T)$ can be rewritten as $N_{T}\left(E_{n}(T)+\right.$ $s / \bar{d})-N_{T}\left(E_{n}(T)\right) \geq k$, we can substitute the argument of the step-function. Upon replacing the sum by an integral over $I(E ; \hbar)$ with weight $d\left(E^{\prime}\right)$ which, in turn, we can asymptotically substitute by $d_{T}\left(E^{\prime}\right):=\mathrm{d} N_{T}\left(E^{\prime}\right) / \mathrm{d} E^{\prime}$ we obtain

$$
\begin{aligned}
& I(k, s ; T) \sim \\
& \quad\left\langle\Theta\left(N_{T}\left(E^{\prime}+\frac{s}{\bar{d}}\right)-N_{T}\left(E^{\prime}\right)-k-1\right) \frac{d_{T}\left(E^{\prime}\right)}{\bar{d}}\right\rangle .
\end{aligned}
$$

The brackets denote the energy average $\langle\ldots\rangle:=$ $\frac{1}{2 \hbar \omega} \int_{E-\hbar \omega}^{E+\hbar \omega} \ldots \mathrm{d} E^{\prime}$, due to which we may asymptotically replace $d_{T}\left(E^{\prime}\right) / \bar{d}$ by 1 . Since the semiclassical limit $\hbar \rightarrow 0$ now implies $\bar{d} \rightarrow \infty$, we have $s / \bar{d} \ll 1$, and expanding the mean part of $N_{T}$ about $E$ yields the periodic-orbit formula

$$
I(k, s ; T) \sim\left\langle\Theta\left(s-k-1+N_{T}^{\mathrm{f}}\left(E^{\prime}+\frac{s}{\bar{d}}\right)-N_{T}^{\mathrm{f}}\left(E^{\prime}\right)\right)\right\rangle
$$

for the integrated level spacing distribution. A similar expression can be obtained for the level spacing distribution $P(k, s ; T)$ itself by taking the derivative of (5). Here we again neglect $d_{T}^{\mathrm{f}}\left(E^{\prime}+s / \bar{d}\right) / \bar{d}$ because of the energy 
average, i.e.

$P(k, s ; T) \sim\left\langle\delta\left(s-k-1+N_{T}^{\mathrm{fl}}\left(E^{\prime}+\frac{s}{\bar{d}}\right)-N_{T}^{\mathrm{fl}}\left(E^{\prime}\right)\right)\right\rangle$.

For the case $k=0$, i.e. for nearest-neighbor level spacings, we will now test these formulae numerically for two toy models. Our first example is a classically integrable system, namely a rectangular quantum billiard with aspect ratio $2 /(1+\sqrt{5})$ and Neumann boundary conditions. For scaling systems the semiclassical limit $\hbar \rightarrow 0$ can be replaced by the high energy limit $E \rightarrow \infty$, with a suitable change in the energy average. For the plots we have taken into account energies in the interval $E \in[0,4000]$ (corresponding to 214 eigenvalues). Figure 11 shows the integrated level spacing distribution for this system where for the cut-off time $T$ we have chosen $T=T_{H}:=2 \pi \hbar \bar{d}$ (dashed-dotted line) and $T=50 T_{H}$ (dashed line), respectively. When increasing the cut-off time $T$ we observe convergence towards the quantum mechanical result (solid line) which in turn in the limit $E \rightarrow \infty$ converges to a Poissonian level spacing distribution (dotted line), cf. [11]

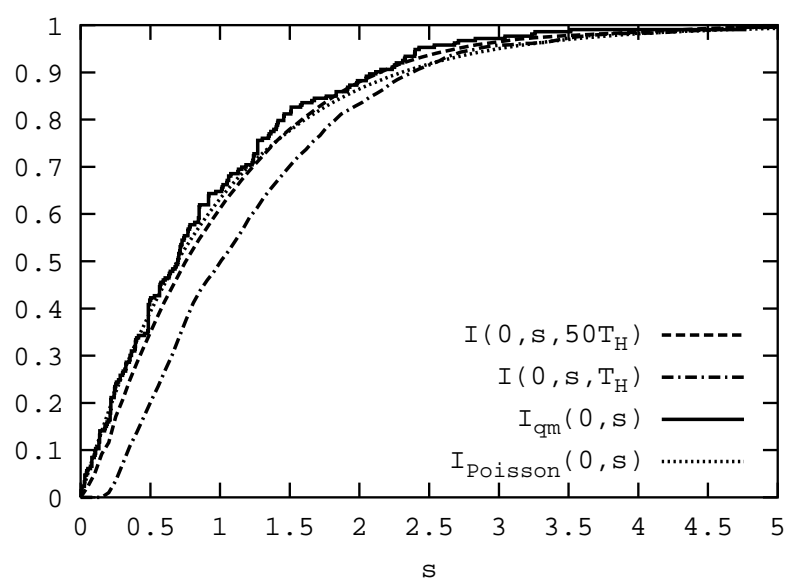

FIG. 1. Integrated level spacing distribution from periodic orbits (5) for a rectangular billiard with cut-off time $50 T_{H}$ (dashed), and $T_{H}$ (dashed-dotted). The quantum mechanical level spacing distribution (solid) and the graph for a Poisson process (dotted) are shown for comparison.

For a second test we have chosen the imaginary parts of the non-trivial zeros of the Riemann zeta function. These serve as a model for quantum chaos, since their spectral correlations are well described by the Gaussian unitary ensemble (GUE), see, e.g., 12 and references therein. The density of the Riemann zeros is related to a sum over primes, see, e.g., [13], in a similar way as the density of states of a quantum system is related to a sum over periodic orbits of the corresponding classical system (11). However, for chaotic systems as well as for the Riemann zeta function it has been observed that there might be an optimal cut-off for the periodic orbit sum, i.e. including arbitrarily long orbits doesn't necessarily improve the result. In the case of the Riemann zeta function this is known as the Riemann-Siegel cut-off [14] which corresponds to $T_{H} / 2$ [15].

In figure 2 the integrated level spacing distribution of the first 649 Riemann zeros with imaginary parts between 0 and 1000 (solid line) is shown as well as the analogue of (5) (dashed line) with a truncation of the sum over primes at the Riemann-Siegel cut-off. We observe a good agreement of both curves at large values of $s$ were both approach the GUE result (dotted line).

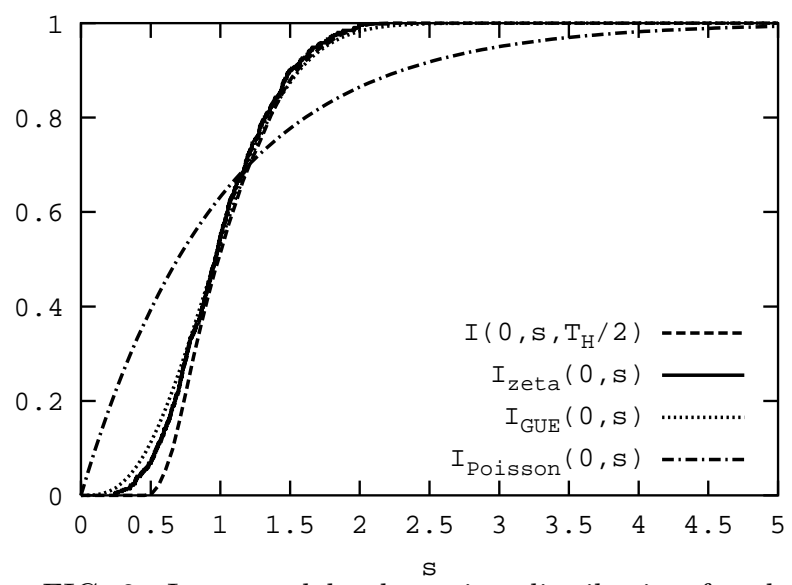

FIG. 2. Integrated level spacing distribution for the zeros of the Riemann zeta function (solid) and the spacing distribution calculated from prime numbers (dashed), cf. (5), $T=T_{H} / 2$. The spacing distribution of the GUE (dotted) and for a Poisson process (dashed-dotted) are shown for comparison.

However, for values of $s$ below 1 the periodic-orbit formula differs from both, the GUE and the exact curve. This is due to the fact that the periodic orbit sum (1), truncated at the order of the Heisenberg time, cannot reproduce features below the scale of mean level spacing. Unlike in the integrable case, we cannot simply increase the cut-off since now the sum over primes behaves like an asymptotically divergent series, i.e. we observe best agreement when truncating the sum at the Riemann-Siegel cut-off. Analogously we will adopt the same point of view for periodic-orbit sums of classically chaotic systems by treating the respective trace formulae like asymptotically divergent series, choosing $T$ of the order of the Heisenberg time.

We remark that in numerical calculations it is necessary to use a smoothed version of the periodic orbit sum (11). We have chosen to add a so-called remainder term as introduced by Aurich and Steiner [6], which in the case of the Riemann zeta function is related to Guinand's formula [16].

Now that we have checked the periodic orbit formula numerically, we attempt to evaluate $P(k, s ; T)$ asymptot- 
ically for generic quantum systems with chaotic classical limit. A well established conjecture on global eigenvalue statistics 17 states that (after suitable normalization) in the semiclassical limit the value distribution of $N_{T}^{\mathrm{fl}}$ is given by a Gaussian with zero mean. In analogy to [8] we also assume Gaussian behavior for the difference $N_{T}^{\mathrm{fl}}\left(E^{\prime}+s / \bar{d}\right)-N_{T}^{\mathrm{fl}}\left(E^{\prime}\right)$. Although this assumption leads to the correct result in the case of two-point correlations [8,18, we remark that it corresponds to neglecting crosscorrelations between $N_{T}^{\mathrm{fl}}$ at different arguments, which, especially for small $s$, will become important. Thus, in the limit $s \rightarrow \infty, s / \bar{d} \rightarrow 0$, the energy average in (6) can be performed approximately, yielding

$$
P(k, s ; T) \approx \frac{1}{\sqrt{2 \pi \sigma^{2}}} \exp \left(-\frac{(s-k-1)^{2}}{2 \sigma^{2}}\right),
$$

where the $\left(s\right.$-dependent) variance $\sigma^{2}:=\left\langle\left(N_{T}^{\mathrm{fl}}\left(E^{\prime}+s / \bar{d}\right)-\right.\right.$ $\left.\left.N_{T}^{\mathrm{fl}}\left(E^{\prime}\right)\right)^{2}\right\rangle$ of the Gaussian is still to be determined. This last step can be done by substituting the trace formula (1) for $N_{T}^{\mathrm{f}}$, employing an expansion in $s / \bar{d}$ (cf. [18]) and making use of the diagonal approximation [5, 4 ,

$$
\begin{aligned}
\sigma^{2} & \sim\left(\sum_{\left|r T_{\gamma}\right| \leq T} \sum^{\prime} \frac{\mathcal{A}_{\gamma r}}{2 \pi \mathrm{i} r} \mathrm{e}^{\frac{\mathrm{i}}{\hbar} r S_{\gamma}}\left(\mathrm{e}^{\frac{\mathrm{i}}{\hbar} r T_{\gamma} \frac{s}{d}}-1\right)\right)^{2} \\
& \approx \sum_{\left|r T_{\gamma}\right| \leq T} \sum_{\leq}^{\prime} \frac{g\left|\mathcal{A}_{\gamma r}\right|^{2}}{(2 \pi r)^{2}}\left(2-2 \cos \left(\frac{s r T_{\gamma}}{\hbar \bar{d}}\right)\right),
\end{aligned}
$$

where $g$ denotes the generic multiplicity of orbits which share the same action, cf. [4, 10. Using the classical sum rule [5] we obtain

$\sigma^{2} \approx \frac{g}{\pi^{2}} \int_{0}^{T} \frac{1-\cos \left(\frac{s T^{\prime}}{\hbar \bar{d}}\right)}{T^{\prime}} \mathrm{d} T^{\prime} \sim \frac{g}{\pi^{2}}\left(\log \left(\frac{s T}{\hbar \bar{d}}\right)+\gamma\right)$,

$\gamma$ denoting Euler's constant. We remark that the subleading term of this expansion is not unique at this point, since it is influenced by corrections to the sum rule, which unfortunately are unknown, as well as by the choice of the cut-off time. Therefore, when now setting $T=C T_{H}=C 2 \pi \hbar \bar{d}$ we obtain

$$
\sigma^{2} \approx \frac{g}{\pi^{2}}(\log s+\alpha)
$$

Here $\alpha$ is kept as a free parameter, which, however, will not influence the large- $s$ asymptotics. Upon substituting (11) into (7) we obtain a semiclassical formula for the level spacing distributions as $s \rightarrow \infty$,

$$
P_{\mathrm{sc}}(k, s) \approx \sqrt{\frac{\pi /(2 g)}{\log s+\alpha}} \exp \left(-\frac{\pi^{2}(s-k-1)^{2}}{2 g(\log s+\alpha)}\right) .
$$

Since (12) is mainly supported around $s \approx k+1$ we may for a moment substitute $\log s$ by $\log (k+1)$ for large $k$.
The formula obtained that way is consistent with a conjecture for $P(k, s)$ from RMT [19], see also [20], which can be adopted to generic quantum systems with chaotic classical limit 21. However for small $k$ and in particular for nearest-neighbor level spacings (12) fails to reproduce the RMT results. This has to be understood in the following sense: The techniques used are essentially restricted to two-point correlations. Since the mean distance between $x_{n+k+1}$ and $x_{n}$ is given by $k+1$, the $P(k, s)$ can only be dominated by two-point correlations on scales where $s \approx k+1$. Thus an asymptotic evaluation as $s \rightarrow \infty$ also implies large $k$.

We will now investigate the relation of our result (12) with the method of Bogomolny and Keating for the twopoint correlation function $R_{2}(s)$, which can be represented as the sum

$$
R_{2}(s):=\sum_{k=1}^{\infty} P(k, s)-1 .
$$

Substituting (12) for the level spacing distributions and applying the Poisson summation formula yields

$$
\begin{aligned}
R_{2, \mathrm{sc}}(s) \approx \sum_{\nu \in \mathbb{Z} \backslash\{0\}} & \frac{1}{2} \exp \left(-2 \pi^{2} \nu^{2} \sigma^{2}\right) \exp (2 \pi \mathrm{i} \nu s) \\
\times & {\left[1+\operatorname{erf}\left(\frac{s-1+2 \pi \mathrm{i} \nu \sigma^{2}}{\sqrt{2} \sigma}\right)\right], }
\end{aligned}
$$

where $\sigma^{2}$ is given by (11). The leading terms as $s \rightarrow \infty$ derive from $\nu= \pm 1$ (as can be easily seen from the first exponential), i.e.

$$
R_{2, \mathrm{sc}}(s) \approx 2 \mathrm{e}^{-2 g \alpha} \frac{\cos (2 \pi s)}{s^{2 g}} .
$$

Comparing with the large- $s$ asymptotics of two-point correlation functions of RMT we observe that we have obtained the leading oscillatory contribution, which can be expected from the method of Bogomolny and Keating [8,18, but are missing the term which corresponds to the diagonal approximation of the spectral form factor, cf. [4, 8, 22]. The reason for this is that between (4) and (6) we have neglected the term $d_{T}^{\mathrm{fl}}\left(E^{\prime}\right) d_{T}^{\mathrm{fl}}\left(E^{\prime}+s / d\right) / \bar{d}^{2}$, which there was consistent, since it can be checked that doing so does not change the large- $s$ asymptotics of (12). However, in the sum (13) the diagonal approximation of this term yields the missing term of $R_{2}(s)$.

In this sense our result for the level spacing distributions (for large $k$ ) is in leading order consistent with RMT two-point correlations. Thus, we can now compare (15) to the respective results from RMT, in order to fix $\alpha$. For systems without time-reversal symmetry $(g=1)$ we have to compare with the GUE result, see, e.g. [23], thus obtaining $\alpha=\log (2 \pi)$. Analogously for time reversal invariant systems $(g=2)$ we obtain $\alpha=\log (\sqrt{2} \pi)$ by comparing with GOE two-point correlations. Thus, with 
the substitution $\log s \approx \log (k+1)$ for large $k$ we have obtained Gaussians for the $P(k, s)$ with approximate variances

$$
\sigma^{2}(k) \approx \frac{g}{\pi^{2}}(\log (k+1)+\alpha) .
$$

We briefly remark that the reasoning easily carries over to the third universality class, the Gaussian symplectic ensemble (GSE). To this end we have to consider trace formulae for particles with half integer spin [24,25]. For time reversal invariant systems one has to take into account Kramers' degeneracy as in 18]. Again using the Gaussian ansatz (7) and methods of [10] for the calculation of $\sigma^{2}$ we obtain

$$
P_{\mathrm{spin}}(k, s) \approx \sqrt{\frac{\pi}{\log s+\alpha}} \exp \left(-\frac{\pi^{2}(s-k-1)^{2}}{\log s+\alpha}\right),
$$

where the same procedure as above yields $\alpha=\log 8$.

Summarizing, we have derived periodic orbit formulae for the level spacing distributions $P(k, s)$ and $I(k, s)$. Numerical tests show that using purely classical input even the nearest-neighbor spacing distribution can be obtained for both, classically integrable and chaotic systems, where in the latter case we cannot go below the scale of mean level spacing. In a second part we have presented an asymptotic evaluation of the formulae for large spacings which yields good approximations, if we restrict ourselves to the case of large $k$, but does not apply to the nearest-neighbor level spacing.

It is a pleasure to acknowledge stimulating discussions with Dr. Jens Bolte, Prof. Jon Keating, Roman Schubert and Prof. F. Steiner. This work was supported by Deutsche Forschungsgemeinschaft (DFG) under contract no. STE 241/10-1.

* E-mail address: kep@physik.uni-ulm.de

[1] M. V. Berry and M. Tabor: Level clustering in the regular spectrum, Proc. R. Soc. London Ser. A 356 (1977) 375-394.

[2] O. Bohigas, M.-J. Giannoni and C. Schmit: Characterization of chaotic quantum spectra and universality of level fluctuation laws, Phys. Rev. Lett. 52 (1984) 1-4.

[3] M. C. Gutzwiller: Periodic Orbits and Classical Quantization Conditions, J. Math. Phys. 12 (1971) 343-358.

[4] M. V. Berry: Semiclassical theory of spectral rigidity, Proc. R. Soc. London Ser. A 400 (1985) 229-251.

[5] J. H. Hannay and A. M. Ozorio de Almeida: Periodic orbits and a correlation function for the semiclassical density of states, J. Phys. A 17 (1984) 3429-3440.
[6] R. Aurich and F. Steiner: Staircase functions, spectral rigidity, and a rule for quantizing chaos, Phys. Rev. A 45 (1992) 583-592.

[7] R. Aurich, C. Matthies, M. Sieber and F. Steiner: Novel Rule for Quantizing Chaos, Phys. Rev. Lett. 68 (1992) 1629-1632.

[8] E. B. Bogomolny and J. P. Keating: Gutzwiller's Trace Formula and Spectral Statistics: Beyond the Diagonal Approximation, Phys. Rev. Lett. 77 (1996) 1472-1475.

[9] M. V. Berry and M. Tabor: Closed orbits and the regular bound spectrum, Proc. R. Soc. London Ser. A 349 (1976) 101-123.

[10] J. Bolte and S. Keppeler: Semiclassical form factor for chaotic systems with spin 1/2, J. Phys. A 32 (1999) 8863-8880.

[11] Z. Cheng and J. L. Lebowitz: Statistics of energy levels in integrable quantum systems, Phys. Rev. A 44 (1991) R3399-R3402.

[12] M. V. Berry and J. P. Keating: The Riemann zeros and eigenvalue asymptotics, SIAM Rev. 41 (1999) 236-266.

[13] E. C. Titchmarsh: The theory of the Riemann zetafunction, The Clarendon Press Oxford University Press, New York, 2nd edn., (1986), Edited and with a preface by D. R. Heath-Brown.

[14] C. L. Siegel: Über Riemanns Nachlaß zur analytischen Zahlentheorie, Quellen und Studien zur Geschichte der Math., Astr. und Physik 2 (1932) 45-80.

[15] M. V. Berry and J. P. Keating: A rule for quantizing chaos?, J. Phys. A 23 (1990) 4839-4849.

[16] A. P. Guinand: A summation formula in the theory of prime numbers, Proc. London Math. Soc. (2) 50 (1948) $107-119$.

[17] R. Aurich, J. Bolte and F. Steiner: Universal Signatures of Quantum Chaos, Phys. Rev. Lett. 73 (1994) 13561359 .

[18] S. Keppeler: Two-point correlations of the Gaussian symplectic ensemble from periodic orbits, J. Phys. A 33 (2000) L503-L507.

[19] J. B. French, P. A. Mello and A. Pandey: Statistical Properties of Many-Particle Spectra. II. Two-Point Correlations and Fluctuations, Ann. Phys. (NY) 113 (1978) 277-293.

[20] T. A. Brody, J. Flores, J. B. French, P. A. Mello, A. Pandey and S. S. M. Wong: Random-matrix physics: spectrum and strength fluctuations, Rev. Modern Phys. 53 (1981) 385-479.

[21] R. Aurich and F. Steiner: Energy-Level Statistics of the Hadamard-Gutzwiller Ensemble, Physica D 43 (1990) 155-180.

[22] F. Haake: Quantum Signatures of Chaos, SpringerVerlag, Berlin Heidelberg, (2000).

[23] M. L. Mehta: Random Matrices, Academic Press, San Diego, 2nd edn., (1991).

[24] J. Bolte and S. Keppeler: Semiclassical Time Evolution and Trace Formula for Relativistic Spin-1/2 Particles, Phys. Rev. Lett. 81 (1998) 1987-1991.

[25] J. Bolte and S. Keppeler: A semiclassical approach to the Dirac equation, Ann. Phys. (NY) 274 (1999) 125-162. 